\title{
Suicide among North Carolina women, 1989-93: information from two data sources
}

\author{
C W Runyan, K E Moracco, L Dulli, J Butts
}

Injury Prevention 2003;9:67-72

See end of article for authors' affiliations

Correspondence to: Dr Carol W Runyan, CB 7505 Chase Hall, University of North Carolina, Chapel Hill, NC 27599-7505, USA: carol_runyan@unc.edu

\begin{abstract}
Objectives: To characterize the events and examine suicide precursors among women and to examine gaps in surveillance.

Setting: A statewide study in North Carolina.

Methods: Suicides of women age 15 and older for the time period 1989-93, as identified from the Office of the Chief Medical Examiner, were included. All case files were reviewed by hand and telephone interviews were attempted with investigating law enforcement officials for every case in 1993. Results: Altogether 882 suicides met the case definition, for an age adjusted rate that fluctuated between 5.53 and 7.26 per 100000 women across the period. Interviews with law enforcement officials were completed for 135 of the 177 cases from 1993. White women had rates nearly three times those of racial minorities. Women under age 45 were proportionally more likely than older women to have recently experienced the breakup of an intimate relationship. Information about precursors was not as consistently reported as had been hoped. Medical examiner records were variable in completeness. Law enforcement interviews frequently did not yield information about the factors we had hoped to examine, probably because the investigations were conducted primarily to rule out homicide.

Conclusions: This study suggests somewhat different precursor patterns by age group. It also points to the need for reconsidering how suicide surveillance is accomplished as a strategy to guide intervention.
\end{abstract}

S icide accounts for nearly 6000 deaths among women ges 15 and older in the US each year, making it the second leading cause of injury death among women. ${ }^{1-3}$ Rates have remained relatively stable over the past two decades, decreasing from 5.84 per 100000 in 1981 to 4.20 per 100000 in $1998 .^{1}$

Previous studies of suicide among women have identified substantial differences in rates by race/ethnicity and age, with non-Hispanic white women having a rate twice that of other racial/ethnic groups and women between the ages of 35 and 59 experiencing the highest rates. ${ }^{1}$ Though there appear to be no major differences by marital status, being employed outside the home may be protective. ${ }^{4-6}$

Not surprisingly, mental illness, including major depression, has been repeatedly found to be a common precursor as have other forms of psychological problems and hopelessness, panic attacks and post-traumatic stress disorder. ${ }^{7-12}$ Both prior sexual assault and intimate partner violence have been implicated as precursors of suicide attempts..$^{92-14}$ Bailey et al found that the risk of suicide was five times greater in homes where the victim had ready access to a gun. ${ }^{7}$

Death investigation reports (for example, medical examiner records) are a logical source of information for studying suicide, yet several studies indicate the limitations in data contained in these reports with respect to case identification, classification, and/or record keeping practices. ${ }^{8}{ }^{15-17}$ No report could be found based on the use of law enforcement records or interviews as a means of investigating suicide.

This population based study, one of the largest to date, was designed to identify cases of suicide among women in North Carolina and explore the circumstances surrounding these deaths. In particular, we were interested in exploring the possible role of intimate partner violence as a precursor. In addition, this study explored two data sources, medical examiner case files and interviews with law enforcement officers with respect to documentation of precursors to suicide.

\section{METHODS}

We identified decedents from the North Carolina Office of the Chief Medical Examiner (OCME) database then gathered information from two sources: (A) hard copy reports filed by the OCME and (B) telephone interviews with law enforcement officers.

According to state law, every suicide or other injury death must be investigated by a medical examiner at the county level. Reports are sent to the OCME where they are reviewed and entered into a statewide database. The case files are stored at the OCME, and contain the investigation report, death certificate, autopsy and toxicology reports, and occasionally newspaper clippings and correspondence.

Using the OCME database, we identified all cases in which the manner of death was classified as suicide, the victim was a woman age 15 or older, and the fatal injury was inflicted in North Carolina between 1 January 1989 and 31 December 1993. Research assistants reviewed all of the case files, abstracting and coding information using a standard protocol.

For those cases that occurred during 1993, trained interviewers conducted 15-20 minute telephone interviews with the primary investigating law enforcement officers assigned to each case to gather information about the decedent, the context of the suicide, including whether there was a known history of intimate partner violence. These interviews took place between May and August, 1996. Calls were preceded by a letter to the Chief or Sheriff of the investigating law enforcement agency that indicated our intention to call, noted the names of victims in cases for which we would be seeking information, and suggested that the investigating officer review the file in advance of the call.

We used published population data from the Census Bureau for each of the study years to calculate suicide rates per 100000 women 15 and older, and age adjusted rates by the direct method of standardization using the North Carolina 1990 population as the standard. 
Table 1 Characteristics of the suicide event, North Carolina females ages 15+, 1989-93 ( $n=882$ )

\begin{tabular}{|c|c|c|c|c|c|c|c|c|}
\hline \multirow[b]{2}{*}{ Characteristic } & \multicolumn{7}{|c|}{ Percentage by age group } & \multirow[b]{2}{*}{$\begin{array}{l}\text { Total } \\
\left(n=870^{*}\right)\end{array}$} \\
\hline & $\begin{array}{l}15-24 \\
(n=104)\end{array}$ & $\begin{array}{l}25-34 \\
(n=169)\end{array}$ & $\begin{array}{l}35-44 \\
(n=180)\end{array}$ & $\begin{array}{l}45-54 \\
(n=159)\end{array}$ & $\begin{array}{l}55-64 \\
(n=131)\end{array}$ & $\begin{array}{l}65-74 \\
(n=92)\end{array}$ & $\begin{array}{l}75+ \\
(n=35)\end{array}$ & \\
\hline \multicolumn{9}{|l|}{ Method used } \\
\hline Handgun & 40.4 & 44.4 & 37.2 & 29.6 & 43.5 & 48.9 & 42.8 & 40.0 \\
\hline Prescription drugs & 21.2 & 23.1 & 30.0 & 35.8 & 26.0 & 18.6 & 5.7 & 25.9 \\
\hline Other firearm & 13.5 & 11.2 & 11.1 & 5.7 & 6.1 & 5.4 & 2.9 & 8.7 \\
\hline Non-prescription drugs & 3.8 & 3.0 & 2.8 & 5.7 & 1.5 & 4.3 & 0.0 & 3.4 \\
\hline Carbon monoxide & 9.6 & 4.1 & 4.4 & 6.9 & 5.3 & 8.7 & 0.0 & 5.8 \\
\hline Sharp instrument & 1.9 & 1.8 & 1.1 & 1.3 & 1.5 & 2.2 & 8.6 & 1.8 \\
\hline Force (strangulation, suffocation, etc) & 7.7 & 7.1 & 6.7 & 5.7 & 8.4 & 7.6 & 31.4 & 8.0 \\
\hline Other & 1.9 & 5.3 & 6.7 & 9.3 & 7.7 & 4.3 & 8.6 & 6.4 \\
\hline \multicolumn{9}{|l|}{ Murder-suicide event } \\
\hline Yes & 0.0 & 0.6 & 3.3 & 0.6 & 1.5 & 1.1 & 0.0 & 1.3 \\
\hline No/unknown/missing & 100.0 & 99.4 & 96.7 & 99.4 & 98.5 & 98.9 & 100.0 & 98.7 \\
\hline \multicolumn{9}{|l|}{ Suicide in a motor vehicle } \\
\hline Yes & 11.7 & 8.3 & 10.2 & 10.1 & 5.5 & 5.4 & 0.0 & 8.3 \\
\hline No & 85.4 & 89.9 & 89.3 & 89.9 & 91.4 & 93.5 & 100.0 & 90.3 \\
\hline Unknown/missing & 2.9 & 1.8 & 0.5 & 0.0 & 3.1 & 1.1 & 0.0 & 1.4 \\
\hline \multicolumn{9}{|l|}{ Owner of the weapon used } \\
\hline Victim & 1.9 & 1.8 & 2.2 & 3.8 & 3.8 & 12.1 & 6.1 & 3.8 \\
\hline Family member of victim & 3.9 & 2.4 & 0.6 & 0.6 & 3.8 & 4.4 & 0.0 & 2.2 \\
\hline Friend of victim & 0.0 & 1.2 & 1.1 & 0.0 & 0.8 & 1.1 & 3.0 & 0.8 \\
\hline Other & 4.8 & 1.8 & 4.5 & 1.9 & 0.8 & 1.1 & 0.0 & 2.4 \\
\hline No weapon used & 45.2 & 45.2 & 52.2 & 61.0 & 46.5 & 41.8 & 45.4 & 49.4 \\
\hline Unknown & 44.2 & 47.6 & 39.4 & 32.7 & 44.3 & 39.5 & 45.5 & 41.4 \\
\hline \multicolumn{9}{|l|}{ Suicide note left } \\
\hline Yes & 34.6 & 29.6 & 31.8 & 26.4 & 29.8 & 22.8 & 48.6 & 30.2 \\
\hline No & 50.0 & 47.3 & 48.1 & 49.7 & 52.7 & 57.6 & 37.1 & 49.7 \\
\hline Unknown/missing & 15.4 & 23.1 & 20.1 & 23.9 & 17.5 & 19.6 & 14.3 & 20.1 \\
\hline \multicolumn{9}{|l|}{ Time of event } \\
\hline 24:00-5:59 & 14.4 & 13.0 & 8.9 & 7.4 & 6.1 & 10.9 & 2.9 & 9.7 \\
\hline $6-11: 59$ & 9.6 & 9.5 & 17.2 & 16.5 & 18.3 & 18.5 & 28.5 & 15.4 \\
\hline $12: 00-17: 59$ & 28.9 & 26.0 & 19.4 & 17.0 & 23.7 & 16.3 & 14.3 & 21.5 \\
\hline $18: 00-23: 59$ & 14.4 & 21.3 & 15.0 & 14.5 & 9.2 & 8.7 & 5.7 & 14.1 \\
\hline Unknown/missing & 32.7 & 30.2 & 39.4 & 44.6 & 42.7 & 45.6 & 48.6 & 39.3 \\
\hline
\end{tabular}

\section{RESULTS}

\section{Characteristics of decedents}

We identified a total of 882 eligible female suicides, 177 of which occurred in 1993. The overall age adjusted rate fluctuated between 5.53 and 7.26 per 100000 women across the five year period. Decedents ranged in age from 15 to 89 , with a median age of 41 . White women, who comprised nearly $90 \%$ of the victims, were over-represented, and had rates ( 7.38 per 100000$)$ that were three times higher than those for African-American women (2.49 per 100 000). Among those women over age 19, 48.9\% were married, $20.1 \%$ were separated or divorced, $14.2 \%$ were widowed, and $16 \%$ had never married. More than three quarters of the women over age $19(79.9 \%)$ had at least a high school education and a third (33.6\%) had some post-secondary training. Among women of all ages, $24.7 \%$ of the victims had positive blood alcohol levels, $17.1 \%$ of whom had levels exceeding $80 \mathrm{mg} / \mathrm{dl}$ (or $0.08 \%$ by volume). OCME records indicated that $299(34.4 \%)$ of the women had made previous suicide attempts or had a known history of suicidal ideation.

\section{Event characteristics}

In nine cases, the suicide was associated with other deaths including homicides $(n=8)$ and/or another suicide $(n=2)$. Firearms, $82 \%$ of which were handguns, were used in $40 \%$ of the suicides (table 1). The next largest category of method was poisoning by prescription drugs $(25.9 \%)$. The OCME reports indicated that suicide notes were present in $30.2 \%$ of the cases.

From the law enforcement interviews concerning those cases that occurred in 1993, we learned that $74 \%$ of the 135 women for whom a location of injury was known killed themselves in their own homes, or in the homes of family or friends.

\section{Precursors of the suicides}

As noted in table 2, most of the OCME records contained no mention of a history of chronic conditions, addictions, or child abuse. However, for those precursors recorded, we found differences by age group. Evidence of chronic health problems was recorded as present in the medical examiner record for fewer than $20 \%$ of the women under age 45 , more than $40 \%$ of those in the 55-74 age group, and over three quarters of those age 75 or older. This includes chronic diseases (for example, diabetes, heart disease, cancer) as well as mental or physical disabilities. Across all age groups, approximately $10 \%$ of women were noted as having a history of substance abuse problems, including alcoholism.

A history of depression or some other mental health problem was the most frequent $(62.2 \%)$ precursor noted in the medical examiner data for the female suicides occurring in 1989-93. Over a third $(34.4 \%)$ of the women had previous suicide attempts or ideation. Though only $3.5 \%$ of the OCME files noted a history of domestic violence, being involved in an interpersonal conflict was noted in $17.7 \%$ of the cases overall, and $33.6 \%$ of the cases among women 15-24. In addition interpersonal conflict was the second most common precursor noted by law enforcement officers (37.8\%) for all the 1993 cases (table 3). In 76\% (39 of 51) of these 1993 cases where such a conflict was noted, the conflict occurred between the victim and her current or former intimate partner. Other frequent precursors noted by the law enforcement officers for the 1993 cases included previous suicide attempts or ideation $(40.0 \%)$, a history of mental illness $(36.3 \%)$, an intimate relationship ending or recently ended (32.6\%), and a chronic disease or disability (17.0\%) (table 3$)$. 
Table 2 Precursors of female suicide in North Carolina from OCME data, 1989-93 ( $n=882$ )

\begin{tabular}{|c|c|c|c|c|c|c|c|c|}
\hline \multirow[b]{2}{*}{ Precursor } & \multicolumn{7}{|c|}{ Percentage by age group } & \multirow[b]{2}{*}{$\begin{array}{l}\text { Total } \\
(n=870 *)\end{array}$} \\
\hline & $\begin{array}{l}15-24 \\
(n=104)\end{array}$ & $\begin{array}{l}25-34 \\
(n=169)\end{array}$ & $\begin{array}{l}35-44 \\
(n=180)\end{array}$ & $\begin{array}{l}45-54 \\
(n=159)\end{array}$ & $\begin{array}{l}55-64 \\
(n=131)\end{array}$ & $\begin{array}{l}65-74 \\
(n=92)\end{array}$ & $\begin{array}{l}75+ \\
(n=35)\end{array}$ & \\
\hline \multicolumn{9}{|l|}{ History of mental problems } \\
\hline Yes & 46.2 & 58.0 & 62.8 & 72.3 & 64.9 & 69.6 & 51.4 & 62.2 \\
\hline No/unknown/missing & 53.8 & 42.0 & 37.2 & 27.7 & 35.1 & 30.4 & 48.6 & 37.8 \\
\hline \multicolumn{9}{|l|}{ Chronic disease or disability } \\
\hline Yes & 7.7 & 10.7 & 17.2 & 30.8 & 43.9 & 45.7 & 77.1 & 26.7 \\
\hline No/unknown/missing & 92.3 & 89.3 & 82.8 & 69.2 & 56.1 & 54.3 & 22.9 & 73.3 \\
\hline \multicolumn{9}{|c|}{ Prior suicide attempt or ideation } \\
\hline Yes & 29.8 & 36.7 & 40.6 & 30.2 & 31.3 & 35.9 & 31.4 & 34.4 \\
\hline No/unknown/missing & 70.2 & 63.3 & 59.4 & 69.8 & 68.7 & 64.1 & 68.6 & 65.6 \\
\hline \multicolumn{9}{|l|}{ History of alcohol problems } \\
\hline Yes & 8.7 & 10.1 & 12.2 & 15.1 & 6.1 & 6.5 & 0.0 & 9.9 \\
\hline No/unknown/missing & 91.3 & 89.9 & 87.8 & 84.9 & 93.9 & 93.5 & 100.0 & 90.1 \\
\hline \multicolumn{9}{|l|}{ Addiction history or drug use } \\
\hline Yes & 17.3 & 16.0 & 8.3 & 10.1 & 5.3 & 9.8 & 8.6 & 10.9 \\
\hline No/unknown/missing & 82.7 & 84.0 & 91.7 & 89.9 & 94.7 & 90.2 & 91.4 & 89.1 \\
\hline \multicolumn{9}{|l|}{ History of domestic abuse } \\
\hline Yes & 4.8 & 4.7 & 5.0 & 3.8 & 1.5 & 0.0 & 0.0 & 3.5 \\
\hline No/unknown/missing & 95.2 & 95.3 & 95.5 & 96.2 & 98.5 & 100.0 & 100.0 & 96.5 \\
\hline \multicolumn{9}{|c|}{ Involved in interpersonal conflict } \\
\hline Yes & 33.6 & 24.3 & 21.1 & 17.6 & 6.9 & 2.2 & 2.9 & 17.7 \\
\hline No/unknown/missing & 66.4 & 76.7 & 78.9 & 82.4 & 93.1 & 97.8 & 97.1 & 82.3 \\
\hline \multicolumn{9}{|l|}{ Financial problems } \\
\hline Yes & 3.9 & 2.4 & 2.2 & 1.9 & 3.9 & 2.8 & 0.0 & 2.5 \\
\hline No/unknown/missing & 96.1 & 97.6 & 97.8 & 98.1 & 96.1 & 97.8 & 100.0 & 97.5 \\
\hline \multicolumn{9}{|c|}{ Intimate relationship ending/recently ended } \\
\hline Yes & 8.6 & 10.7 & 7.8 & 5.8 & 0.8 & 0.0 & 0.0 & 5.9 \\
\hline No/unknown/missing & 91.4 & 89.3 & 92.2 & 94.2 & 99.2 & 100.0 & 100.0 & 94.1 \\
\hline \multicolumn{9}{|c|}{ Job loss/unemployment/recently retired } \\
\hline Yes & 1.0 & 5.3 & 3.9 & 2.5 & 3.9 & 8.7 & 11.4 & 4.4 \\
\hline No/unknown/missing & 99.0 & 94.7 & 96.1 & 97.5 & 96.1 & 91.3 & 88.6 & 95.6 \\
\hline \multicolumn{9}{|c|}{ History of suicide in family/friends } \\
\hline Yes & 3.9 & 0.6 & 2.2 & 0.6 & 3.9 & 0.0 & 2.9 & 1.9 \\
\hline No/unknown/missing & 93.1 & 99.4 & 97.8 & 99.4 & 96.1 & 100.0 & 97.1 & 98.1 \\
\hline \multicolumn{9}{|c|}{ Diagnosis or concern regarding fatal disease } \\
\hline Yes & 0.0 & 0.0 & 0.6 & 1.9 & 0.0 & 3.3 & 5.7 & 1.0 \\
\hline No/unknown/missing & 100.0 & 100.0 & 99.4 & 98.1 & 100.0 & 97.7 & 94.3 & 99.0 \\
\hline \multicolumn{9}{|c|}{ Problems at school (age $\leqslant 24$ years) } \\
\hline Yes & 1.0 & NA & NA & NA & NA & N.A & NA & NA \\
\hline No/unknown/missing & 99.0 & & & & & & & \\
\hline \multicolumn{9}{|l|}{ History of child abuse } \\
\hline Yes & 1.0 & 0.0 & 0.6 & 0.0 & 0.0 & 0.0 & 0.0 & 0.2 \\
\hline No/unknown/missing & 99.0 & 100.0 & 99.4 & 100.0 & 100.0 & 100.0 & 100.0 & 99.8 \\
\hline \multicolumn{9}{|l|}{ Under job related stress } \\
\hline Yes & 0.0 & 1.8 & 3.9 & 3.2 & 1.6 & 0.0 & 0.0 & 2.0 \\
\hline No/unknown/missing & 100.0 & 98.2 & 96.1 & 96.8 & 98.4 & 100.0 & 100.0 & 98.0 \\
\hline \multicolumn{9}{|l|}{ Victim recently arrested } \\
\hline Yes & 1.9 & 2.4 & 2.8 & 0.6 & 0.8 & 0.0 & 0.0 & 1.5 \\
\hline No/unknown/missing & 98.1 & 97.6 & 97.2 & 99.4 & 99.2 & 100.0 & 100.0 & 98.5 \\
\hline
\end{tabular}

\section{Information available in the data sources}

For 135 of the 177 cases $(76.3 \%)$ that occurred in 1993, we completed interviews with law enforcement officers. We were unable to obtain information from the law enforcement agencies for 34 of the remaining 42 cases because the agencies either did not have records or did not investigate the deaths $(n=25)$, or because we were unable to contact the investigating law enforcement agencies after multiple attempts $(\mathrm{n}=9)$. For the eight remaining cases, we were unable to obtain information because law enforcement had initially ruled the deaths as due to causes other than suicide, including one ruled as a homicide, two ruled as accidental deaths, and five considered by the law enforcement officials to be deaths due to "natural causes". Although the OCME later reclassified each of these eight cases as suicides, they had not been investigated as such by law enforcement officials.

As we have done in prior research on femicide, we intended to supplement the data available from the OCME with data collected through interviews with the law enforcement officers concerning all the cases of female suicide. ${ }^{18}$ Unlike the femicide investigations, however, law enforcement interviews about the suicides yielded limited additional information. So, we halted data collection after conducting interviews about the cases occurring in 1993.

Some information, such as whether the decedent had a history of mental health problems or left a suicide note was recorded frequently in the medical examiner files or noted by the officers we interviewed. In contrast, other information that might help to understand the context of the suicides is not recorded often, including information about a history of drug or alcohol problems, history of child or adult traumas (for example, abuse, sexual assault), or information about other precipitating factors (for example, recent job stress, history of domestic violence, or interpersonal conflict).

\section{DISCUSSION}

Although this is one of the largest population based studies of female suicide to date using routine surveillance systems, we discovered that existing data sources are less complete than we had hoped. Though it is not possible to determine how 
Table 3 Precursors of female suicide in North Carolina from law enforcement interview data, 1993 ( $n=135$ )

\begin{tabular}{|c|c|c|c|c|c|c|c|c|}
\hline \multirow[b]{2}{*}{ Precursor } & \multicolumn{7}{|c|}{ Percentage by age group } & \multirow[b]{2}{*}{$\begin{array}{l}\text { Total } \\
(n=135)\end{array}$} \\
\hline & $\begin{array}{l}15-24 \\
(n=21)\end{array}$ & $\begin{array}{l}25-34 \\
(n=20)\end{array}$ & $\begin{array}{l}35-44 \\
(n=34)\end{array}$ & $\begin{array}{l}45-54 \\
(n=22)\end{array}$ & $\begin{array}{l}55-64 \\
(n=17)\end{array}$ & $\begin{array}{l}65-74 \\
(n=12)\end{array}$ & $75+(n=9)$ & \\
\hline \multicolumn{9}{|l|}{ History of mental problems } \\
\hline Yes & 19.0 & 35.0 & 38.2 & 54.5 & 41.2 & 33.3 & 22.2 & 36.3 \\
\hline No & 62.0 & 55.0 & 55.9 & 40.9 & 47.0 & 50.0 & 66.7 & 53.3 \\
\hline Unknown/not available/not investigated & 19.0 & 10.0 & 5.9 & 4.6 & 11.8 & 16.7 & 11.1 & 10.4 \\
\hline \multicolumn{9}{|l|}{ Chronic disease or disability } \\
\hline Yes & 0.0 & 15.0 & 14.7 & 4.5 & 35.3 & 33.3 & 44.5 & 17.0 \\
\hline No & 90.5 & 75.0 & 85.3 & 90.9 & 64.7 & 58.3 & 33.3 & 77.0 \\
\hline Unknown/not available/not investigated & 9.5 & 10.0 & 0.0 & 4.6 & 0.0 & 8.4 & 22.2 & 6.0 \\
\hline \multicolumn{9}{|l|}{ Prior suicide attempt or ideation } \\
\hline Yes & 38.1 & 40.0 & 44.1 & 36.4 & 53.0 & 41.7 & 11.1 & 40.0 \\
\hline No & 23.8 & 20.0 & 29.4 & 45.4 & 23.5 & 41.7 & 66.7 & 32.6 \\
\hline Unknown/not available/not investigated & 38.1 & 40.0 & 26.5 & 18.2 & 23.5 & 16.6 & 22.2 & 27.4 \\
\hline \multicolumn{9}{|l|}{ History of alcohol problems } \\
\hline Yes & 14.3 & 20.0 & 29.4 & 27.3 & 23.5 & 8.3 & 0.0 & 20.7 \\
\hline No & 47.6 & 45.0 & 26.5 & 18.2 & 41.2 & 50.0 & 44.4 & 36.3 \\
\hline Unknown/not available/not investigated & 38.1 & 35.0 & 44.1 & 54.5 & 35.3 & 41.7 & 55.6 & 43.0 \\
\hline \multicolumn{9}{|l|}{ Addiction history or drug use } \\
\hline Yes & 9.5 & 5.0 & 2.9 & 13.6 & 5.9 & 0.0 & 0.0 & 5.9 \\
\hline No & 42.9 & 25.0 & 35.3 & 27.3 & 35.3 & 50.0 & 22.2 & 34.1 \\
\hline Unknown/not available/not investigated & 47.6 & 70.0 & 61.8 & 59.1 & 58.8 & 50.0 & 77.8 & 60.0 \\
\hline \multicolumn{9}{|l|}{ History of domestic abuse } \\
\hline Yes & 4.8 & 20.0 & 8.8 & 9.1 & 5.9 & 16.7 & 0.0 & 9.6 \\
\hline No & 85.7 & 65.0 & 85.3 & 86.4 & 94.1 & 75.0 & 100.0 & 83.7 \\
\hline Unknown/not available/not investigated & 9.5 & 15.0 & 5.9 & 4.5 & 0.0 & 8.3 & 0.0 & 6.7 \\
\hline \multicolumn{9}{|l|}{ Involved in interpersonal conflict } \\
\hline Yes & 52.4 & 30.0 & 58.8 & 31.8 & 35.3 & 8.3 & 0.0 & 37.8 \\
\hline No & 19.0 & 35.0 & 11.8 & 31.8 & 47.1 & 41.7 & 77.8 & 31.1 \\
\hline Unknown/not available/not investigated & 28.6 & 35.0 & 29.4 & 36.4 & 17.6 & 50.0 & 22.2 & 31.1 \\
\hline \multicolumn{9}{|l|}{ Financial problems } \\
\hline Yes & 9.5 & 5.0 & 2.9 & 13.6 & 5.9 & 0.0 & 0.0 & 5.9 \\
\hline No & 42.9 & 25.0 & 35.3 & 27.3 & 35.3 & 50.0 & 22.2 & 34.1 \\
\hline Unknown/not available/not investigated & 47.6 & 70.0 & 61.8 & 59.1 & 58.8 & 50.0 & 77.8 & 60.0 \\
\hline \multicolumn{9}{|l|}{ Intimate relationship ending/recently ended } \\
\hline Yes & 42.8 & 35.0 & 47.1 & 36.4 & 23.5 & 0.0 & 0.0 & 32.6 \\
\hline No & 28.6 & 35.0 & 29.4 & 45.4 & 58.8 & 50.0 & 88.9 & 42.2 \\
\hline Unknown/not available/not investigated & 28.6 & 30.0 & 23.5 & 18.2 & 17.7 & 50.0 & 11.1 & 25.2 \\
\hline Job loss/unemployment/recently retired & & & & & & & & \\
\hline Yes & 9.5 & 5.0 & 11.8 & 18.2 & 17.7 & 41.7 & 22.2 & 15.6 \\
\hline No & 81.0 & 80.0 & 82.3 & 54.5 & 58.8 & 33.3 & 66.7 & 68.8 \\
\hline Unknown/not available/not investigated & 9.5 & 15.0 & 5.9 & 27.3 & 23.5 & 25.0 & 11.1 & 15.6 \\
\hline History of suicide in family/friends & & & & & & & & \\
\hline Yes & 4.8 & 5.0 & 2.9 & 0.0 & 5.9 & 0.0 & 0.0 & 3.0 \\
\hline No & 47.6 & 35.0 & 47.1 & 45.5 & 58.8 & 41.7 & 55.6 & 46.7 \\
\hline Unknown/not available/not investigated & 47.6 & 60.0 & 50.0 & 54.5 & 35.3 & 58.3 & 44.4 & 50.3 \\
\hline Diagnosis or concern regarding fatal diseas & & & & & & & & \\
\hline Yes & 0.0 & 5.0 & 3.0 & 4.6 & 11.8 & 41.7 & 22.2 & 8.9 \\
\hline No & 85.7 & 65.0 & 79.4 & 81.8 & 64.7 & 74.7 & 77.8 & 73.3 \\
\hline Unknown/not available/not investigated & 14.3 & 30.0 & 17.6 & 13.6 & 23.5 & 16.6 & 0.0 & 17.8 \\
\hline Problems at school (age $\leqslant 24$ years) & & & & & & & & \\
\hline Yes & 14.3 & NA & NA & NA & NA & NA & NA & NA \\
\hline No & 57.1 & & & & & & & \\
\hline Unknown/not available/not investigated & 28.6 & & & & & & & \\
\hline History of child abuse & & & & & & & & \\
\hline Yes & 4.8 & 0.0 & 0.0 & 0.0 & 5.9 & 0.0 & 0.0 & 1.5 \\
\hline No & 23.8 & 10.0 & 11.8 & 4.5 & 17.6 & 16.7 & 0.0 & 12.6 \\
\hline Unknown/not available/not investigated & 71.4 & 90.0 & 88.2 & 95.5 & 76.5 & 83.3 & 100.0 & 85.9 \\
\hline Under job related stress & & & & & & & & \\
\hline Yes & 0.0 & 25.0 & 20.6 & 9.1 & 5.9 & 0.0 & 0.0 & 11.1 \\
\hline No & 71.4 & 50.0 & 47.1 & 54.5 & 70.6 & 91.7 & 88.9 & 62.2 \\
\hline Unknown/not available/not investigated & 28.6 & 25.0 & 32.3 & 36.4 & 23.5 & 8.3 & 11.1 & 26.7 \\
\hline Victim recently arrested & & & & & & & & \\
\hline Yes & 9.5 & 0.0 & 0.0 & 0.0 & 0.0 & 0.0 & 0.0 & 1.5 \\
\hline No & 66.7 & 80.0 & 85.3 & 95.4 & 94.1 & 100.0 & 100.0 & 86.7 \\
\hline Unknown/not available/not investigated & 23.8 & 20.0 & 14.7 & 4.6 & 5.9 & 0.0 & 0.0 & 11.8 \\
\hline
\end{tabular}

many cases of suicide are missed by the medical examiner system, we have no reason to believe that large numbers of cases are missed or that case identification is systematically biased. However, information contained in the case files is variable. For the most part, data about the decedent, including a toxicology report, and means of death is present. However, less detailed information is available about precursors or circumstances surrounding the suicides, limiting the utility of the data for intervention development. Most of the information we were seeking about precursor information is not specifically requested on the standard OCME report form, but rather must be abstracted from information mentioned voluntarily in the narrative section. Some of the information about medical history (for example, history of substance 
Box 1: Objectives recommended by the US National Council for Suicide Prevention, 2001 ${ }^{21}$

- Objective 1 1. 1: By 2005, develop and refine standardized protocols for death scene investigations and implement these protocols in counties (or comparable jurisdictions).

- Objective 11.2: By 2005, increase the proportion of jurisdictions that regularly collect and provide information for followback studies on suicides.

- Objective 11.3: By 2005, increase the proportion of hospitals (including emergency departments) that collect uniform and reliable data on suicidal behavior by coding external cause of injuries utilizing the categories included in the International Classification of Diseases.

- Objective 11.4: By 2005, implement a national violent death reporting system that includes suicides and collects information not currently available from death certificates.

- Objective 11.5: By 2005, increase the number of states that produce annual reports on suicide and suicide attempts, integrating data from multiple states' data management systems

- Objective 11.6: By 2005, increase the number of nationally representative surveys that include questions on suicidal behavior.

- Objective 11.7: By 2005, implement pilot projects in several states that link and analyze information related to self destructive behavior derived from separate data systems, including for example law enforcement, emergency medical services, and hospitals.

abuse or depression) is included in a check box section, However, failure to check the box may mean absence of the condition or merely that the information was not discovered or not recorded. In addition, given the current lack of specific mandates to report this type of information in either law enforcement or medical examiner systems, the gaps in records are not surprising. Hence, for the data shown in table 2, it is impossible to differentiate the missing and unknown values from a "no". In contrast, the data in table 3 are derived from the interviews with law enforcement officers who were queried specifically about each item listed.

In our previous research on femicide, we found that interviews with law enforcement officers yielded a wealth of information concerning the context of the femicide, as well as the events that led up to it. ${ }^{18}$ However, in the course of applying a similar method to female suicide, we found that the practice of most law enforcement agencies when investigating suicides is to rule out homicide rather than to determine and document precursors to the suicide. Consequently, for purposes of epidemiologic surveillance or research on suicide, this source is less valuable than we had expected.

From the data available, however, our study is consistent with prior research suggesting that a history of mental illness and previous suicide attempts and ideation are risk factors for suicide. Given the prevalence estimate that 6.7 million US women are afflicted with major depressive disorders each year, the potential for suicide is a significant public health issue. ${ }^{19}$ Most of the suicides in our series were committed with either firearms or prescription medications, take place at the woman's home, and rarely indicate that domestic violence was a precursor. Additionally, certain suicide precursors, such as chronic illness or disability and interpersonal conflicts vary by the victim's age, while others, such as a history of mental illness and previous suicidal behavior, are more consistent across age groups. This suggests the need for closer attention to designing interventions appropriate to the circumstances of specific age groups.

To aid in the prevention of suicide, as with any health problem, accurate and complete surveillance data are required. ${ }^{2021}$ Our research suggests the need for more detailed and consistent recording of data on suicide by investigating authorities

\section{Key points}

- Suicide among North Carolina women is an important injury problem, with a rate as high as 7.26 per 100000 in this study period.

- Patterns of suicide among women appear to vary by age group, signaling the need for age specific intervention approaches.

- Consideration should be given to implementing a suicide review process to assist in structuring appropriate interventions.

Routine surveillance of suicide events needs improvement through greater standardization in data collection, drawing on suggestions included in this paper.

- At present, law enforcement investigations do not provide adequate information about suicides.

operating under both law enforcement and public health jurisdictions. This recommendation was recently included in the new report, Reducing suicide - a national imperative, produced by the National Academy of Sciences. ${ }^{22}$ The data elements included in this study (tables 2 and 3) were gleaned from previous research on completed and attempted suicide of men and women, and may be helpful to include in such investigations. Further research should examine how useful such data are in assessing the epidemiology of suicide and guiding development of preventive interventions, and should consider improvements in public health surveillance systems to monitor suicides. Indeed, the US Department of Health and Human Services published a report on suicide prevention strategies in 2001 noting, as we found, that no one source of data provides adequate information on precursors to suicide that could inform suicide prevention efforts. As a result, the report outlined seven specific objectives for improving suicide surveillance, as listed in box 1 . Among other things, the report called for the establishment of a national violent death reporting system, suicide included, that would consist of information derived from a number of sources, including death certificates, coroner's or medical examiner office data, and law enforcement agencies. ${ }^{21}$ As our research indicates, however, the success of such an approach will depend on the thoroughness of the investigation and the completeness of the reporting of information in the participating data systems. We suggest that investigating units (medical examiners, coroners, law enforcement) adopt a standard protocol for investigation in which the principal risk factor information is collected. Inclusion of the topics listed in tables 2 and 3 provide a good starting point but must be accompanied by training in proper data collection, with recognition that this data collection process will take additional time necessitating ample institutional support.

Consideration also might be given to organizing and evaluating a suicide fatality review process, similar to what has been done in many locales to monitor and learn from child fatalities ${ }^{23}$ - at least for a sample of suicides across varied population groups, if not for all cases. Such reviews could investigate, in addition to the items we include in tables 2 and 3 , other risk or protective factors listed in the Department of Health and Human Services report (for example, exposure to media reports of recent suicides, ease of access to lethal means, barriers to accessing mental health and substance abuse services, stigma perceived in seeking care, cultural and religious beliefs about suicide, presence or absence of social support, and skills in problem solving or conflict resolution). ${ }^{21}$

In the meantime, the existing evidence can be used to address strategies for prevention by ensuring accessible and high quality mental health services in the community. Suicide prevention interventions may also need to be tailored to fit the potential risk factors for women of different ages. In addition, the high proportion of cases involving firearms, particularly 
handguns, and the occurrence of suicides in the home environment signals the need for greater attention to gun availability and storage practices in the home.

\section{ACKNOWLEDGEMENTS}

The authors wish to acknowledge the assistance of Ms Patricia Barnes (OCME) for help in accessing data, as well as Evelyn Falls, Ann Gordon, Alison Hilton, and Ronda Zakocs for their help in abstracting data and/or interviewing law enforcement officers. In addition, we are grateful for comments provided by Tamera Coyne-Beasley and Wanda Hunter on an earlier draft of this manuscript. This work was supported by two grants from the National Center for Injury Prevention and Control to The University of North Carolina Injury Prevention Research Center (R49/CCR410533) and (R49/CCR402444).

\section{Authors' affiliations}

C W Runyan, L Dulli, University of North Carolina Injury Prevention Research Center and Department of Health Behavior and Health Education, School of Public Health, University of North Carolina K E Moracco, University of North Carolina Injury Prevention Research Center, Departments of Health Behavior and Health Education and Maternal and Child Health, School of Public Health, University of North Carolina

J Butts, University of North Carolina Injury Prevention Research Center and North Carolina Office of the Chief Medical Examiner

\section{REFERENCES}

1 National Center for Injury Prevention and Control, Centers for Disease Control. National Center for Health Statistics National Vital Statistics System for numbers of deaths, US Bureau of Census for population estimates. Statistics compiled using WISQARS ${ }^{T M}$ produced by the Office of Statistics and Programming, NCIPC, CDC

2 Schnitzer P, Runyan C. Injuries to women in the United States: an overview. Women and Health 1995:23:9-27.

3 Dannenberg A, Baker S, Li G. Intentional and unintentional injuries in women: an overview. Ann Epidemiol 1994:4:133-9.

4 Kposowa A. Marital status and suicide in the National Longitudinal Mortality Study. J Epidemiol Community Health 2000;54:254-61.

5 Burr J, McCall P, Powell-Griner E. Female labor force participation. Soc Sci Med 1997:44:1847-59.

6 Stack S. Effect of female labor force participation on female suicide attitudes. Death Stud 1996:20:285-91.
7 Bailey J, Kellermann A, Somes G, et al. Risk factors for violent death of women in the home. Arch Intern Med 1997:157:777-82.

8 Olson L, Huyler F, Lynch A, et al. Guns, alcohol, and intimate partner violence: the epidemiology of female suicide in New Mexico. Crisis 1999;20:121-6.

9 Kaslow N, Thompson M, Meadows L, et al. Factors that mediate and moderate the link between partner abuse and suicidal behavior in African American women. J Consult Clin Psychol 1998:66:533-40.

10 Kingree J, Thompson M, Kaslow N. Risk factors for suicide attempts among low-income women with a history of alcohol problems. Addict Behav 1999;24:583-7.

11 Davidson J, Hughes D, George L, et al. The association of sexual assault and attempted suicide within the community. Arch Gen Psychiatry 1996:53:550-5.

12 Thompson $M$, Kaslow N, Kingree J, et al. Partner abuse and posttraumatic stress disorder as risk factors for suicide attempts in a sample of low-income, inner-city women. J Trauma Stress $1999 ; 12: 59-72$

13 Stepakoff S. Effects of sexual victimization on suicidal ideation and behavior in US college women. Suicide Life Threat Behav 1998;28:107-26.

14 Wiederman M, Sansone R, Sansone L. History of trauma and attempted suicide among women in a primary care setting. Violence Vict 1998;13:3-9.

15 Bourque L, Kraus J, Cosand B. Attributes of suicides in females. Suicide Life Threat Behav 1983;13:123-38.

16 Dudley M, Kelk N, Florio T, et al. Coroner's records of rural and non-rural cases of youth suicide in New South Wales. Aust N ZJ Psychiatry 1997;32:242-51.

17 van der Voorde H, Hooft P, Mulkers U. On the influence of data sources in aggregated data studies: a comparative study of suicide information based on death certificates and judicial files. J Epidemiol Community Health 1992;47:73-5.

18 Moracco KE, Runyan CW, Butts JD. Femicide in North Carolina, 1991-1993. Homicide Stud 1998;2:442-6.

19 National Institute of Mental Health. The numbers count: 1998. Report No 01-4584. Bethesda, MD: NIMH, 1 January 2001

20 Teutsch S, Churchill R. Principles and practice of public health surveillance. New York: Oxford University Press, 1994.

21 Department of Health and Human Services. National strategy for suicide prevention: goals and objectives for action. Report No 02NLM:HV 6548.A1. Rockville, MD: US DHHS, Public Health Service, 2001

22 Goldsmith SK, Pellmar TC, Kleinman AM, et al, eds. Reducing suicide-a national imperative. Washington, DC: National Academies Press, 2002.

23 Durfee MG, Gellert GA, Tilton-Durfee D. Origins and clinical relevance of child death review teams. JAMA 1992;267:3172-5. 